\title{
Characterization of large off-axis EUV mirrors with high accuracy reflectometry at PTB
}

Laubis, Christian, Buchholz, Christian, Fischer, Andreas, Plöger, Sven, Scholz, Frank, et al.

Christian Laubis, Christian Buchholz, Andreas Fischer, Sven Plöger, Frank Scholz, Heike Wagner, Frank Scholze, Gerhard Ulm, Hartmut Enkisch, Stephan Müllender, Marco Wedowski, Eric Louis, Erwin Zoethout, "Characterization of large off-axis EUV mirrors with high accuracy reflectometry at PTB," Proc. SPIE 6151, Emerging Lithographic Technologies X, 61510I (23 March 2006); doi: 10.1117/12.656246

Event: SPIE 31st International Symposium on Advanced Lithography, 2006, San Jose, California, United States 


\title{
Characterization of large off-axis EUV mirrors with high accuracy reflectometry at PTB
}

\author{
Christian Laubis*, Christian Buchholz, Andreas Fischer, Sven Plöger, Frank Scholz, Heike Wagner, \\ Frank Scholze, and Gerhard Ulm
}

Physikalisch-Technische Bundesanstalt, Abbestraße 2-12, 10587 Berlin, Germany

Hartmut Enkisch, Stephan Müllender, Marco Wedowski

Carl Zeiss SMT AG, Carl Zeiss Straße 22, 73446 Oberkochen, Germany

Eric Louis, Erwin Zoethout

FOM-Institute for Plasma Physics, Edisonbaan 14, NL-3439 MN Nieuwegein, The Netherlands

\begin{abstract}
CZ SMT AG produced large off-axis EUV mirrors as they are used e.g. in ASML's alpha demo tools, the predecessor for Extreme Ultraviolet Lithography (EUVL) production tools by ASML. The coating development and a large part of the actual coatings were done by the FOM-Institute. The Physikalisch-Technische Bundesanstalt (PTB) operates an EUV reflectometry facility at the electron storage ring BESSY II for at-wavelength metrology of full-size EUVL optics with a weight of up to $50 \mathrm{~kg}$ and a diameter of $550 \mathrm{~mm}$. Critical issues for EUVL mirrors are the high reflectivity close to the theoretical limit, the matching of the period to the operating wavelength of the stepper $(13.5 \mathrm{~nm})$ and the imaging properties of the EUV optics. The full multilayer stack needs to be controlled laterally to such extend that the initial subnanometre surface figure of the substrate is preserved. The so-called added figure error should not exceed $100 \mathrm{pm}$ in order to ensure faultless imaging at $13.5 \mathrm{~nm}$ wavelength. Here, we discuss representative results obtained at large offaxis EUV mirrors. We especially discuss the challenges of measurements at higher local angles of incidence according to the optical design and the accuracy needed in sample alignment for measurement of the coating profiles. PTB has shown excellent reproducibility for measurements of the near normal incidence reflectance of flat homogeneous mirrors over several years. For large off-axis EUV mirrors, measurements have to be done at angles significantly off normal, which dramatically increases the influence of angular alignment errors of the sample on the measured peak wavelength. Furthermore, according to the optical design, these optics have gradients of the coating thickness which require exact knowledge of the measurement position in the mirror coordinates. Extensive studies were done to estimate and validate the uncertainties connected to the sample alignment. Our results clearly show that it is possible to meet and verify the tight specifications for the lateral coating profiles of EUV multilayer mirrors. The non-correctable added figure error is significantly better than required and the overall reflectance of the coatings with a special protective capping layer is $65 \%$.
\end{abstract}

Keywords: Extreme ultraviolet, metrology, lithography, at-wavelength characterization, reflectometry, synchrotron radiation

* Corresponding author: Christian.Laubis@ptb.de, phone +49 306392 5097, fax +49 3063925082

Emerging Lithographic Technologies $\mathrm{X}$, edited by Michael J. Lercel,

Proc. of SPIE Vol. 6151, 61510I, (2006) -0277-786X/06/\$15 - doi: 10.1117/12.656246

Proc. of SPIE Vol. $615161510 \mathrm{l}-1$ 


\section{INTRODUCTION}

Extreme Ultraviolet Lithography (EUVL) is the key to the next generation of computer technology. EUVL imaging requires multilayer mirror optics being manufactured with extreme accuracy to ensure uniform illumination at the wafer plane and optimized throughput. As EUVL matures ${ }^{1,2}$, the requirements for the accuracy of reflectivity and wavelength measurements become tighter. Especially the wavelength for the peak mirror reflectivity should be matched to better than $2.5 \mathrm{pm}$ and the uniformity of reflectance should be better than $0.08 \%^{3}$. In response to this demand, substantial improvements in the total measurement uncertainty and repeatability at the Physikalisch-Technische Bundesanstalt (PTB), Germany's national metrology institute, have been achieved and reported ${ }^{4,5}$. The EUV reflectometer allows the measurement of large mirrors ${ }^{6}$ with a diameter as large as $550 \mathrm{~mm}$, a height of $230 \mathrm{~mm}$, and a weight of up to $50 \mathrm{~kg}^{7}$. We present here work on the alignment of aspheric off-axis mirrors which is crucial to achieve accurate measurement results for wavelength and reflectivity needed for the validation of the coating development for EUV optics ${ }^{8,9}$.

\section{SAMPLE - ALIGNMENT}

The SX700 soft X-ray bending magnet beamline was designed first of all for a parallel beam with a reasonably low spot size. This was driven by the demand for a high angular resolution also in the reflected beam for scatterometry applications. To use this high quality beam for accurate measurements ${ }^{4,10}$, the mirror under test has to be properly aligned. For higher angles of incidence (AOI), the alignment of plane mirrors already requires accurate scales in $\Theta$ and $2 \Theta$ (see Fig. 1 and Fig. 4) and the surface of the sample to coincide with the main axis of the goniometer within a few tenth of a millimetre. For figured mirrors, additional details have to be considered: Finding the center-coordinates is no longer trivial. Also the opening of the beam after reflection on a convex surface is inevitable.

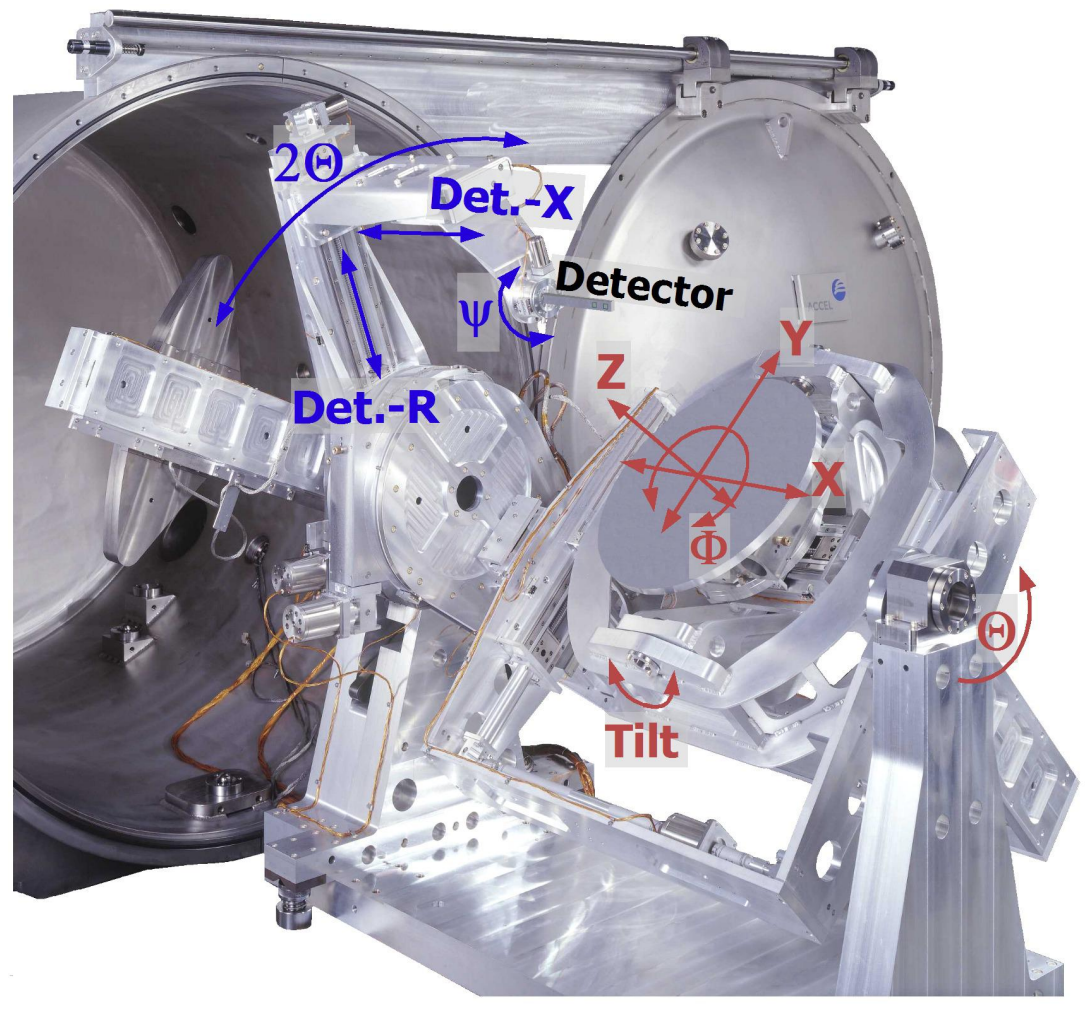

Fig. 1 PTB reflectometer with movements indicated. Red: sample movements, blue: detector movements 
For illustration, Fig. 1 shows the mechanical design of the goniometer with the movements indicated. The sample can be moved in all three orthogonal linear directions and tilted around all of those (red arrows). The detector can be turned to face either the incoming beam or the reflected beam, facilitating reference scans. Also the detector's angle to the beam, the position of the measurement plane and the distance from the sample-surface are adjustable (blue arrows).

2.1.

Plane mirrors

Plane mirrors have been studied at PTB for years with increasing accuracy ${ }^{10,4}$. The adjustment of the center-coordinates is straightforward: Using any reflex from the mirror and moving the $\mathrm{x}$ or $\mathrm{y}$ linear axis, the position of the mirror can directly be determined (Fig. 6, left side). The angular scales however need to be carefully adjusted.

\section{Determination of zero reflection angle}

Our method of measurement involves turning the detector ( $\Psi$-axis in Fig. 1) between direct-beam and reflected-beam measurements ${ }^{7}$. Particularly for the alignment, this approach has strong advantages. Additionally, we save the time and wear to move the whole detector arm all the way from a reflected beam measurement (usually $2 \Theta=3^{\circ}$ for an AOI of $1.5^{\circ}$ ) to a position of $2 \Theta=180^{\circ}$ where the unturned detector would see the beam again. Also the sample would have to be moved out of the beam. We, indeed, rotate the detector using the $\Psi$-axis to look into the direct beam at $2 \Theta=0^{\circ}$, see Fig. 2 , and define the zero angle to be at the position of the incoming beam. A possible offset of the detector switching axis is accounted for.

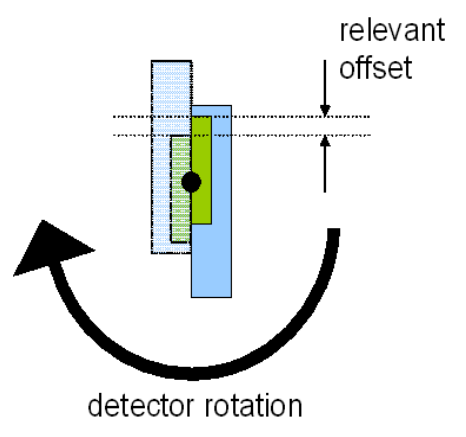

Fig. 2 Determination of zero reflection angle: A $180^{\circ}$ rotation around a pivot point (indicated by the black dot) enables the measurement of direct beam (orientation with bright colours) and reflected beam (transparent). The pivot is designed to be in the center of the surface of the detector, and the offset is measured and corrected.

After defining the zero angle for the detector axis, we define the angle of incidence for the mirror, typically at $1.5^{\circ}$ to the normal. The detector is moved to $3^{\circ}$ and the angle of the sample is scanned to position the beam in the centre of the detector. The main advantage of this approach is illustrated in Fig. 3. Even if the beam is not incident at the correct height, i.e. it does not go through the axis of rotation, the alignment between detector and sample rotation is not disturbed due to the symmetry of the reflection. The angular offset for the reflected beam at the detector is the same for any angle of incidence as well as for the direct beam.
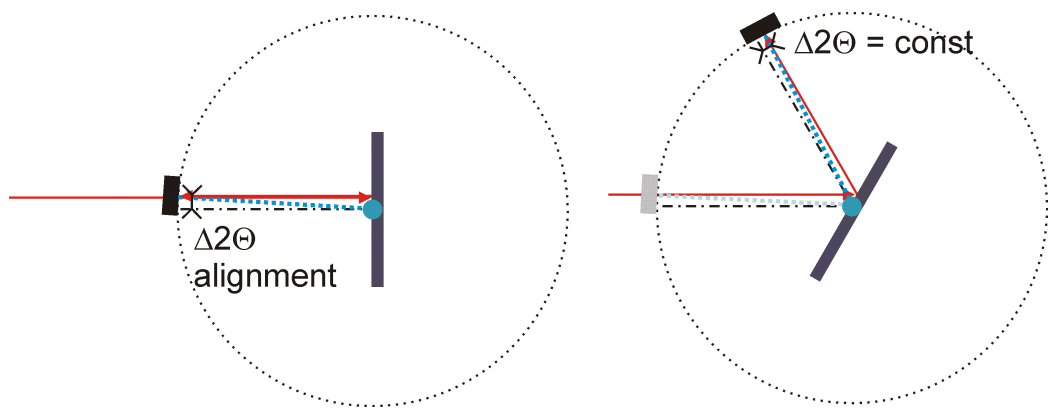

Fig. 3 Scheme of the alignment for the reflection angle. The detector is aligned in the direct beam to define "zero" angle of incidence, left. Due to the symmetry of the reflection geometry, any offset of the beam with respect to the axis of rotation (solid circle in the centre) causes only a fixed offset in detector angle, right. 


\section{Alignment of sample height}

In contrast to the height of the incoming beam which does not effect the determination of the angle of incidence if the axes are properly alignment, as just shown, the surface height of the mirror does. It must coincide with the central axis of the goniometer to assure proper definition of angle of incidence (AOI). The influence of an offset in height $(\Delta z)$ on the AOI is shown in Fig. 4.

With misaligned mirror-height the beam spot will also not longer hit the desired position on the mirror. Especially AOI scans are susceptible to this misalignment. Equation (1) gives the correlation between the angular offset of the detector axis and the offset in the height of the mirror. The designations are taken from Fig. 4.

$\sin (\Delta 2 \Theta)=\frac{\Delta \mathrm{z}}{\mathrm{R}_{\text {Det }}} \frac{\sin (2 * \mathrm{AOI})}{\cos (\mathrm{AOI})}=\frac{2 \Delta \mathrm{z}}{\mathrm{R}_{\text {Det }}} \sin (\mathrm{AOI})$

It is seen that the shift in detector angle, respectively misalignment in AOI, is proportional to $\sin (\mathrm{AOI})$. In order to minimize this misalignment, we always align the AOI at a small angle $\left(1.5^{\circ}\right)$ and use the $\Theta$-axis (which has been qualified with an auto collimation set-up) to go to the desired AOI. Here, the detector-angle $2 \Theta$ is measured for the given angle of the sample to verify the sample height. Any mismatch in detector angle can be converted to a misalignment in sample height. To assure an accuracy of better than $0.02^{\circ}$ in AOI we correct the sample height and repeat the angular alignment. It should be mentioned that any possible offset in incoming photon beam height with respect to the axis of rotation does not compromise this procedure as shown before, see Fig. 3.

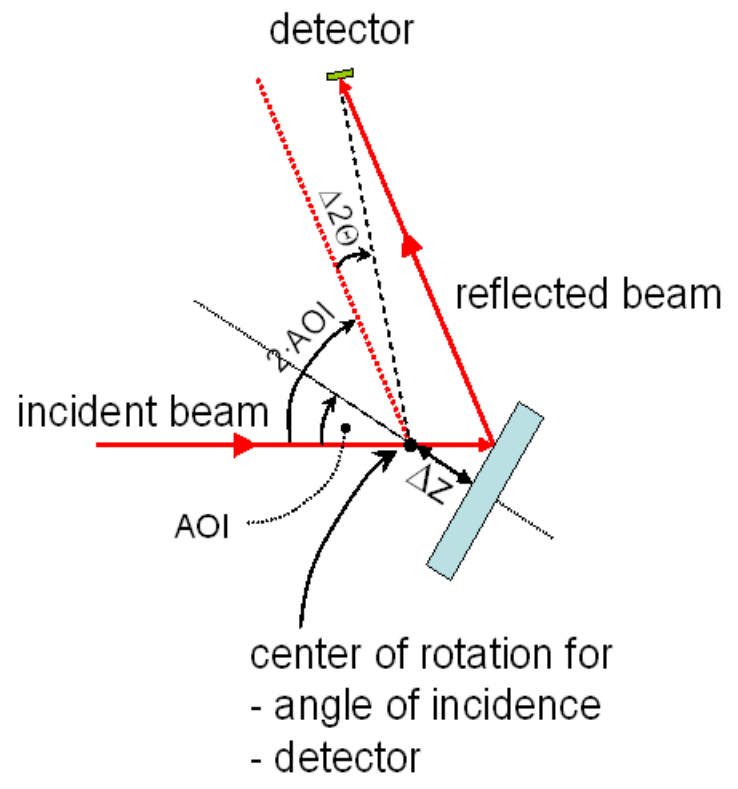

Fig. 4 Visualization of the height adjustment. The mirror surface is not in the center of rotation of the goniometer leading to an inaccurate AOI.

Red arrows symbolize the beam path, the black circle is the main axis $(\Theta)$ of the goniometer. The dashed black line from the main axis to the detector symbolizes the detector-arm, the dotted red line shows the direction of the reflected beam. The difference between the direction of the reflected beam path and the angle of the detectorarm is indicated as $\Delta 2 \Theta$.

$\Delta \mathrm{z}$ is the distance from the mirror surface to the center of rotation.

The centre wavelength $\lambda$ is related to the double-layer thickness $d$ of the coating by the Bragg equation:

$\lambda=2 \mathrm{n}_{\lambda} \mathrm{d} \cos \Theta_{\mathrm{ML}}=\lambda_{0} \cos \Theta_{\mathrm{ML}}$,

with $\mathrm{n}_{\lambda}$ the (average) index of refraction at wavelength $\lambda$, and $\Theta_{\mathrm{ML}}$ the propagation angle in the multilayer coating. Assuming constant $n_{\lambda}$ and $\Theta_{\mathrm{ML}}=\Theta$ we get a cos-dependence for approximation. To keep the shift in measured wavelength, due to the misalignment of the AOI, below $2 \mathrm{pm}$, the angular scales have to be adjusted to better than $0.3^{\circ}$ for measurements close to normal incidence $\left(\mathrm{AOI}=1.5^{\circ}\right)$ and better than $0.02^{\circ}$ for an $\mathrm{AOI}$ of $20^{\circ}$. 
For measuring figured mirrors new challenges that have not been encountered on plane mirrors have to be met. With figured mirrors, there is interdependence between angular and positional adjustment. To tackle these dependencies it is necessary to use well designed adjustment procedures.

\section{Spot size}

As the incident beam at the PTB reflectometry beamline ${ }^{10}$ has a divergence of less than $1 \mathrm{mrad}$, the increase in spot size for plane mirrors over typical detector distances is a few tenths of a millimeter.

For figured mirrors - depending on the curvature of the mirror -, the size of the beam spot after the reflection can differ significantly from the one of the incident beam. Fig. 5 illustrates the situation for a convex mirror.

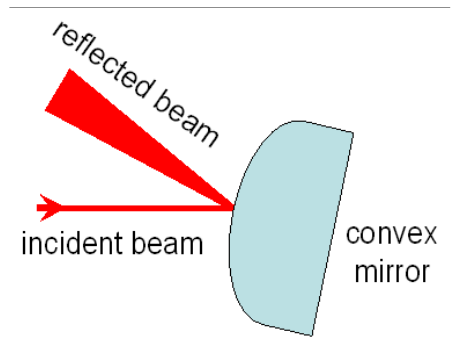

Fig. 5 Visualisation of beam widening by reflection off a convex mirror.

With concave mirrors, the imaging properties of the mirror can be exploited using the detector-distance axis to put the detector at just the right distance to have the same spot size for the incoming and the reflected beam. For convex mirrors there is no such method. The only remedy is to measure as close to the mirror as possible and to use a large detector to capture all reflected intensity. In this case, the uncertainty-limiting factor is the homogeneity of the detector. Therefore we specially select and monitor our detector diodes for homogeneity.

\section{Position on figured mirrors}

Aligning the position on the mirror surface relative to the beam is one necessary step before any measurement can be carried out. For figured mirrors, it is no longer possible to find the edges of the mirror by scanning only the respective linear axis. Fig. 6 visualizes the situation. We have developed two methods to laterally align the position of the mirror.

First - as for plane mirrors - we scan the edges of the substrate, respectively coating, to define the position. Here a linear axis and the corresponding angular axis are moved in parallel. This procedure is particularly suited for (rotational) symmetric mirrors as we actually find the edges of the coating and not of the substrate. The coating, however, may be degraded close to the geometrical edge due to proximity effects in the coater.
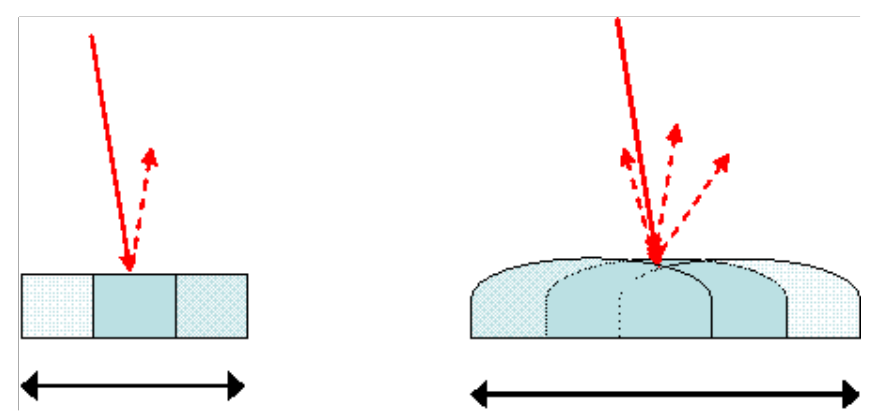

Fig. 6 On plane mirrors (left side) centering the lateral axes is easily accomplished by moving the mirror under the beam. With figured mirrors (right side) the lateral and angular alignment is coupled - requiring a different approach. 
Therefore, we use alignment mirrors as a second method. We place plane mirrors in holders with a well defined edge to touch the side of the mirror to be aligned at defined positions, see Fig. 7. Using this method we only need to determine the positions of the alignment mirrors - without changing the angle of incidence $(\Theta)$ or the tilt. Given the dimensions of the mirror under test, positions on the mirror are then well defined.

\begin{tabular}{c|c|c} 
& edge-adjustment & adjustment mirrors \\
\hline uncertainty in position $\mathrm{x}, \mathrm{y} / \mathrm{mm}$ & 1 & 0.2 \\
\hline${\text { uncertainty in angle } /{ }^{\circ}}$ & $0.02^{*}$ & 0.02
\end{tabular}

Table 1 Comparison of adjustment accuracies for adjustment using substrate edges versus adjustment mirrors.

* for figured mirrors, the uncertainty in position corresponds to an additional uncertainty in AOI depending on the radius of curvature.

\section{MEASUREMENTS ON LARGE OFF-AXIS EUV MIRRORS}

\section{1.}

\section{Lateral alignment}

To align a large off-axis EUV mirror, we use alignment mirrors, see Fig. 7. First, $\Phi$ (rotation around the optical axis) is adjusted using a pair of alignment mirrors mounted against one edge of the sample at the same position in y (and different positions in $\mathrm{x}$ ). Using the $\mathrm{x}$-drive of the goniometer to place these alignment mirrors in the beam, their positions are measured with respect to the $\mathrm{R}$-coordinate of the reflectometer ( $\mathrm{Y}$ in Fig. 1). From this measurement, we obtain the angle between R and the y-axis of the mirror. $\Phi$ is then set to orientate R parallel to $\mathrm{y}$. In this orientation, $\Phi_{0}$, the known position of the alignment mirrors is used to correlate the y-coordinates of the sample to the goniometer R-values. Now, at $\Phi=\Phi_{0}+90^{\circ}$ where $\mathrm{R}$ is parallel to the mirror $\mathrm{x}$-coordinate, we use the third alignment mirror to correlate the $\mathrm{x}$ coordinates of the mirror under test to the goniometer R-values for this value of $\Phi$. Then, we align the angle of incidence in the centre of the mirror under test. Last, we check the height of the mirror. If height-realignment was necessary we iterate the AOI-alignment (as said before). Now in principle, given the shape of the mirror surface, every point on the mirror is known in lateral and angular position of the goniometer coordinates.

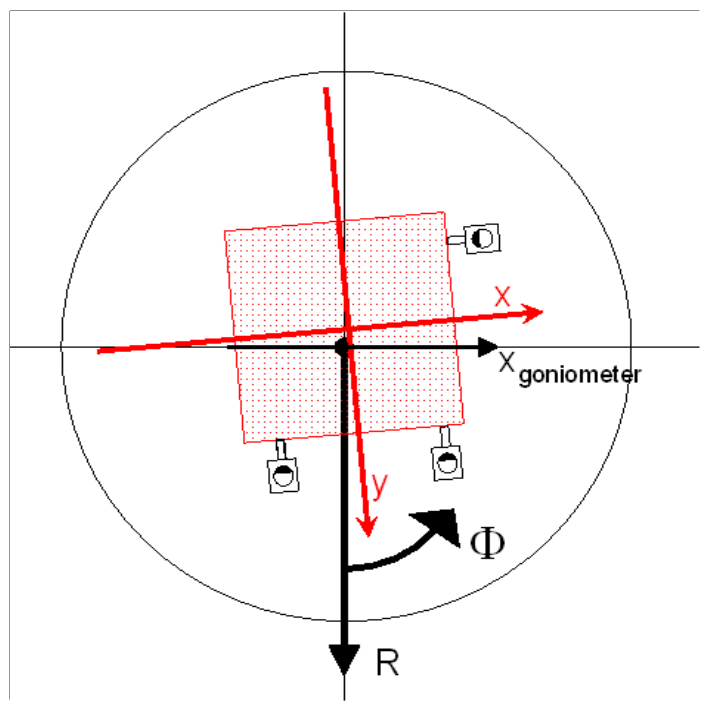

Fig. 7 The different coordinate systems: Mirror sample: $\quad \mathrm{x}$ and $\mathrm{y}$ (in red) Goniometer: $\quad \mathrm{R}, \mathrm{x}$, and $\Phi$ (black) Three alignment mirrors are shown touching the mirror side. 
The range of the R-axis of the sample drive is from $-10 \mathrm{~mm}$ to $290 \mathrm{~mm}$. Therefore we can use the setting $\Phi=\Phi_{1}$ and $\mathrm{R}=\mathrm{x}$ as well as $\Phi=\Phi_{1}+180^{\circ}$ and $\mathrm{R}=$-x, for $|\mathrm{x}|<10$. If everything is well aligned, this is physically the same spot on the sample. For mirrors with a graded coating, therefore, a final test of the alignment can be done; by measuring several points on the mirror surface (sufficiently close the centre) twice. If the mirror were misaligned, the curves measured would not coincide. Particularly for mirrors with a strongly graded coating, this is a sensitive test of the alignment.

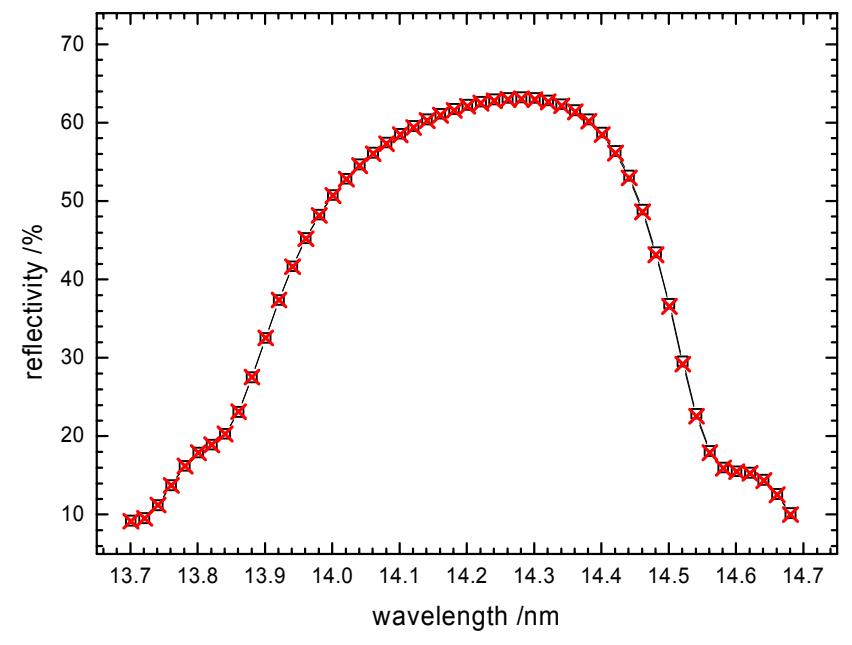

Fig. 8 Alignment test of a mirror with graded coating. The figure shows the measured reflectance on the center spot of the mirror, measured at $\Phi_{0}(\square)$, and with the mirror turned by $180^{\circ}$ around its optical axis (x).

To validate the lateral alignment uncertainty, we used a large off-axis EUV mirrors with a large gradient in its multilayer coating thickness. Fig. 8 gives the data for the test. After aligning all axis as described above the curves shown were measured: $\left(\Phi_{0}, \mathrm{R}=\mathrm{x}\right)$ (black symbols) and $\left(\Phi_{0}+180^{\circ}, \mathrm{R}=-\mathrm{x}\right)$ (red symbols). The value for center-wavelength of these measurements differs by only $0.6 \mathrm{pm}$ which is, however, well beyond our short term repeatability of $0.06 \mathrm{pm}^{4}$. Given the local gradient of the multilayer of about $3 \mathrm{pm} / \mathrm{mm}$, the positional offset between the two measurements shown is $0.2 \mathrm{~mm}$. This is twice the offset in y-alignment due to the rotation by $180^{\circ}$. Therefore, the result verifies the uncertainties of the adjustment method using alignment mirrors.

\subsection{Representative result}

The most critical issue for the multilayer system on the projection optics is the non-correctable added figure error. The wavelength of the mirrors must not only match the desired design wavelength of the EUV optics, it can also be used to determine the multilayer period $\Lambda$ and thus the total film thickness from the determination of the wavelength of maximum reflectance of the reflectivity curves measured at points distributed over the entire surface. As an example of an optical element, which is representative for the projection optics, we will discuss the coating of a concave mirror. The active optical surface has a diameter of $120 \mathrm{~mm}$ and the optical design requires a slightly parabolic profile. Fig. 9 shows the relative thickness profile obtained, determined in two perpendicular directions where both curves nicely coincide, demonstrating the required rotational symmetry of the coating profile. The coating profile can be corrected through lower order Zernike polynomial corrections to determine the non-correctable added figure error. The alignment-adjusted design period $\Lambda_{\mathrm{a}}(\mathrm{r})$ is given by:

$\Lambda_{\mathrm{a}}(\mathrm{r})=\Lambda_{\mathrm{d}}(\mathrm{r})+\mathrm{a}+\mathrm{b}_{\mathrm{x}, \mathrm{y}} \mathrm{r}+\mathrm{cr}^{2}+\mathrm{dr}^{4}$ 
with $\mathrm{r}$ being the radial distance and $\Lambda_{\mathrm{d}}(\mathrm{r})$ the design period. Taking the difference between the measured period $\Lambda$ and the alignment-adjusted design period $\Lambda_{\mathrm{a}}(\mathrm{r})$, the non-correctable added figure error can be calculated. The distribution of this figure error is shown in Fig. 10 and amounts to an rms value of $15 \mathrm{pm}$ only, which is seven times better than specified.

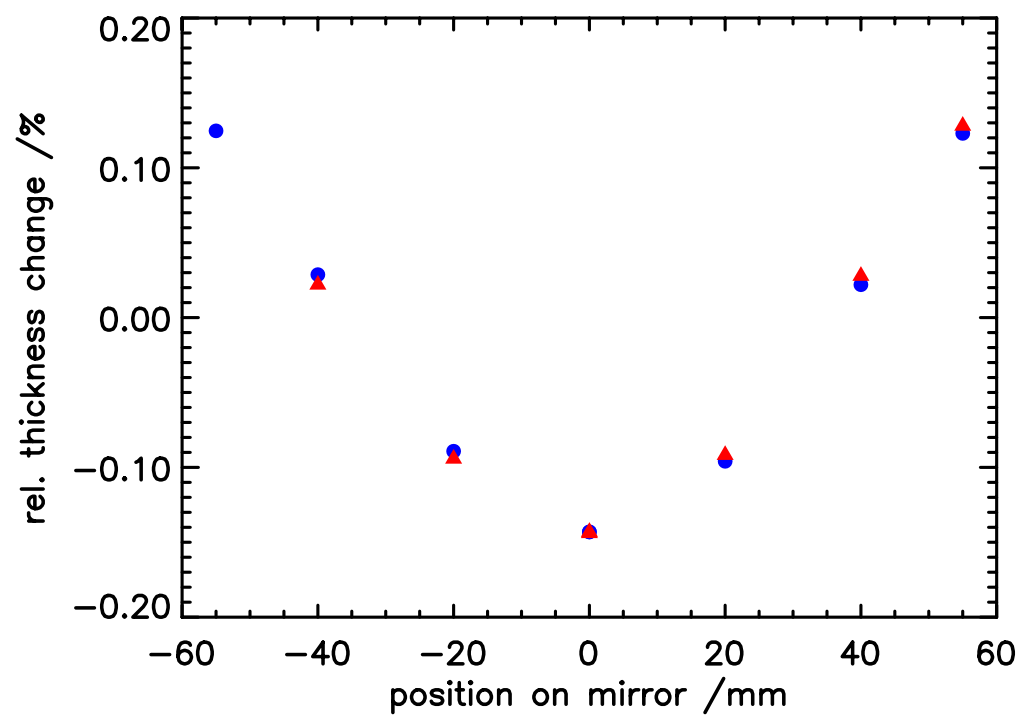

Fig. 9 Relative thickness profile of the coating on a projection optics element, measured in two perpendicular directions.

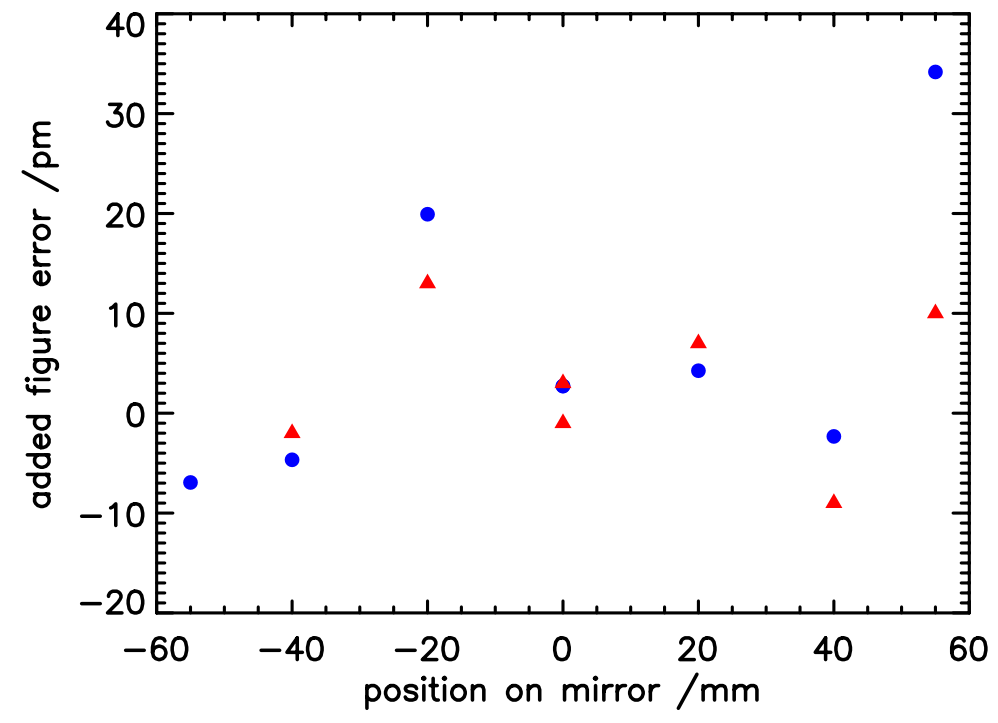

Fig. 10 Non correctable added figure error determined in two perpendicular directions. The rms value amounts to $15 \mathrm{pm}$.

\section{CONCLUSION}

PTB operates a reflectometer for the investigation of samples with a diameter of up to $550 \mathrm{~mm}$, a height of up to $230 \mathrm{~mm}$ and a weight of $50 \mathrm{~kg}$. In response to the needs of the industry, the ability to properly align and measure off-axis aspherical mirrors has been developed and proven. Using alignment mirrors attached to the sample, a lateral positioning uncertainty of $0.2 \mathrm{~mm}$ is achieved. Using a proper procedure for alignment of the sample height with respect to the axis of rotation, it is possible to adjust the AOI to better than $0.02^{\circ}$ in the range from $1.5^{\circ}$ to $20^{\circ}$. We showed that the 
mechanical motions of the EUV reflectometer allow for redundant positioning which can be used to include test and verification measurements in the alignment of large off-axis EUV mirrors on a regular basis. Measurements at PTB were used to validate the multilayer coating development at SMT AG and FOM with regard to the tight specifications. The above discussed example of the performance of multilayer coatings on projection optics clearly show that it is possible to meet the tight specifications of EUV multilayer coatings for first generations of EUVL demonstration systems. On the multilayer systems for the projection optics we demonstrated that the most critical issue, the non-correctable added figure error, can be a factor of seven better than required. The overall reflectance of the coatings that are capped with a special protective layer is $65 \%$. This investigation also showed ways to further reduce PTB's uncertainties to keep up with the future needs of the industry in characterization of EUV optics.

\section{ACKNOWLEDGEMENT}

The authors thank the BESSY staff for reliable operation of the storage ring as an essential prerequisite for high-accuracy measurements. Part of this work was supported by: Bundesministerum für Bildung und Forschung Projekt 13N8088 "Grundlagen der EUV-Lithographie" and MEDEA Project "EXTATIC."

\section{REFERENCES}

1 H. Meiling, V. Banine, P. Kürz, N. Harned, "Progress in the ASML EUV program," Proc. SPIE 5374, 31 - 42 (2004)

2 H. Meiling, V. Banine, N. Harned, B. Blum, P. Kürz, and H. Meijer, "Development of the ASML EUV alpha demo tool," Proc. SPIE 5751, 90-101 (2005)

3 E. Gullikson, S. Mrowka, and B. Kaufmann, "Recent developments in EUV reflectometry at the Advanced Light Source," Proc. SPIE 4343, 363 - 373 (2001)

4 F. Scholze, C. Laubis, C. Buchholz, A. Fischer, S. Plöger, F. Scholz, H. Wagner and G. Ulm, "Status of EUV Reflectometry at PTB", Proc. SPIE 5751, 749-758 (2005)

5 F. Scholze, B. Beckhoff, G. Brandt, R. Fliegauf, A. Gottwald, R. Klein, B. Meyer, U. Schwarz, R. Thornagel, J. Tümmler, K. Vogel, J. Weser, and G. Ulm, "High-accuracy EUV metrology of PTB using synchrotron radiation," Proc. SPIE 4344, 402-413 (2001)

6 U. Dinger, G. Seitz, S. Schulte, F. Eisert, C. Münster, S. Burkart, S. Stacklies, C. Bustaus, H. Höfer, M. Mayer, B. Fellner, O. Hocky, M. Rupp, K. Riedelsheimer, P. Kürz, "Fabrication and metrology of diffraction limited soft x-ray optics for the EUV microlithography," Proc SPIE 5193 18-28 (2004)

7 J. Tümmler, G. Brandt, J. Eden, H. Scherr, F. Scholze, G. Ulm, "Characterization of the PTB EUV reflectometry facility for large EUVL optical components," Proc. SPIE 5037, 265-273 (2003)

8 E. Louis, A. Yakshin, E. Zoethout, R. van de Kruijs, I. Nedelcu, S. van der Westen, T. Tsarfati, F. Bijkerk, H. Enkisch, S. Müllender, B. Wolschrijn, B. Mertens, "Enhanced performance of EUV multilayer coatings," Proc SPIE 5900, 1-4 (2005)

9 E. Louis, E. Zoethout, R. van de Kruijs, I. Nedelcu, A. Yakshin, S. van der Westen, T. Tsarfati, F. Bijkerk, H. Enkisch, S. Müllender, "Multilayer coatings for the EUVL process development tool," Proc SPIE 5751, 1170-1177 (2005)

10 F. Scholze, J. Tümmler, G. Ulm, "High-accuracy radiometry in the EUV range at the PTB soft X-ray radiometry beamline," Metrologia 40, S224-S228 (2003) 